\title{
Anisotropy of Transverse Sound in the Heavy-Fermion Superconductor UPt $_{3}$
}

\author{
B. S. Shivaram, Y. H. Jeong, and T. F. Rosenbaum \\ The James Franck Institute and the Department of Physics, The University of Chicago, Chicago Illinois 60637 \\ and \\ D. G. Hinks \\ Materials Science and Technology Division, Argonne National Laboratories, Argonne, Illinois 60439 \\ (Received 18 December 1985)
}

\begin{abstract}
We report the first measurements of the attenuation of ultrasound in the basal plane of superconducting $\mathrm{UPt}_{3}$. Transverse sound propagating along the $b$ axis shows a marked anisotropy in its temperature dependence when the polarization is rotated in and out of the basal plane. For polarization in the basal plane the attenuation varies linearly with temperature down to $35 \mathrm{mK}$ and the slope scales as the square of the frequency. Our results appear to indicate the presence of an additional attenuation mechanism when compared with recent theories of anisotropic superconductors in the dirty limit.
\end{abstract}

PACS numbers: $74.30 . \mathrm{Gn}, 74.70 . \mathrm{Rv}$

Since the discovery of bulk superconductivity ${ }^{1}$ in $\mathrm{UPt}_{3}$, there has been wide speculation that the Cooper pairs in this heavy-fermion compound are in an oddparity, spin-triplet state. Evidence cited for this assignment includes (i) the presence of a $T^{3} \ln T$ term in the specific heat at low temperatures, ${ }^{1}$ (ii) the observed anisotropy of the upper critical field, ${ }^{2}$ and (iii) the power-law behavior of the attenuation of longitudinal ultrasound. ${ }^{3}$ At low temperatures the ultrasonic attenuation is observed to follow a $T^{2}$ power law which has been attributed to an Anderson-Brinkman-Morel (ABM) type of order parameter, ${ }^{4,5}$ where the gap has zeros at points on the Fermi surface. For a polar state, where there are zeros at lines on the Fermi surface, Pethick and Pines ${ }^{5}$ have recently shown that the ultrasonic attenuation should scale as $T^{2} \ln ^{2} T$ which is experimentally hard to distinguish from a $T^{2}$ dependence. Furthermore, it is well known that a $T^{2}$ dependence is also expected in gapless superconductors with singlet pairing. ${ }^{6}$ Thus, the results from present ultrasonic experiments do not permit one to assign unambiguously a particular structure to the gap in superconducting $\mathrm{UPt}_{3}$. The situation is further complicated by more recent experiments ${ }^{7}$ where the attenuation of ultrasound is claimed to be more appropriately described by a $T^{3}$ rather than a $T^{2}$ power law.

All measurements to date of both longitudinal and transverse ultrasound in superconducting $\mathrm{UPt}_{3}$ have been along the $c$ axis. However, it is useful to carry out the experiments along various crystal directions in order to exploit the power of the ultrasonic method in exploring the anisotropy of the energy gap. In this way, possible effects due to the topology of the Fermi surface may be subtracted out. Furthermore, transverse ultrasound is expected to interact more selectively than longitudinal acoustic waves with the electrons on the Fermi surface, thereby providing a more sensi- tive probe of the energy-gap anisotropy. ${ }^{8}$ It is even predicted that in the case of an ABM type of order parameter there are special directions where the temperature dependence of the transverse ultrasonic attenuation scales ${ }^{9}$ as $T^{4}$ or possibly as $T^{6}$. No such special directions exist for the longitudinal case.

In this Letter we report the measurements of transverse sound propagating along the $b$ axis (in the basal plane) with the polarization, $\hat{\mathbf{e}}$, both parallel and perpendicular to the basal plane. Contrary to previous observations, we find a linear temperature dependence of the attenuation when both $\hat{\mathbf{q}}$ and $\hat{\mathbf{e}}$ are in the basal plane. On the other hand, for ê perpendicular to the basal plane, our results are similar to the work reported previously. ${ }^{3,7}$

Single crystals of $\mathrm{UPt}_{3}$ were grown by the vertical-float-zone refining method. After spark planing and annealing at $1200^{\circ} \mathrm{C}$ for forty hours, two opposite faces of the samples were polished to optical flatness. $20-\mathrm{MHz} A C$-cut quartz transducers bonded by Armstrong C-1 epoxy ${ }^{10}$ were used to propagate and detect transverse ulrasound in the frequency range 20 to $172 \mathrm{MHz}$. A rf heterodyne spectrometer with a phase-sensitive detector was used to measure both the attenuation and the velocity at constant temperature. ${ }^{11}$ Pulse voltages were less than $10 \mathrm{~V}$ peak-to-peak and, with a repetition rate of $100 \mathrm{~Hz}$, did not cause any heating at the lowest temperatures. All measurements were repeated at three different power levels separated by more than an order of magnitude and were found to yield the same results. The samples had $T_{c}=500 \mathrm{mK}$ with a $10 \%-90 \%$ transition width of $50 \mathrm{mK}$.

We show in Fig. 1 the change in attenuation, $\alpha$, along the $b$ axis for $94-\mathrm{MHz}$ transverse sound with the polarization $\hat{\mathbf{e}} \| \hat{\mathbf{a}}$. Three important features in the figure need to be emphasized. First, and most important, the attenuation at the lowest temperatures does not 


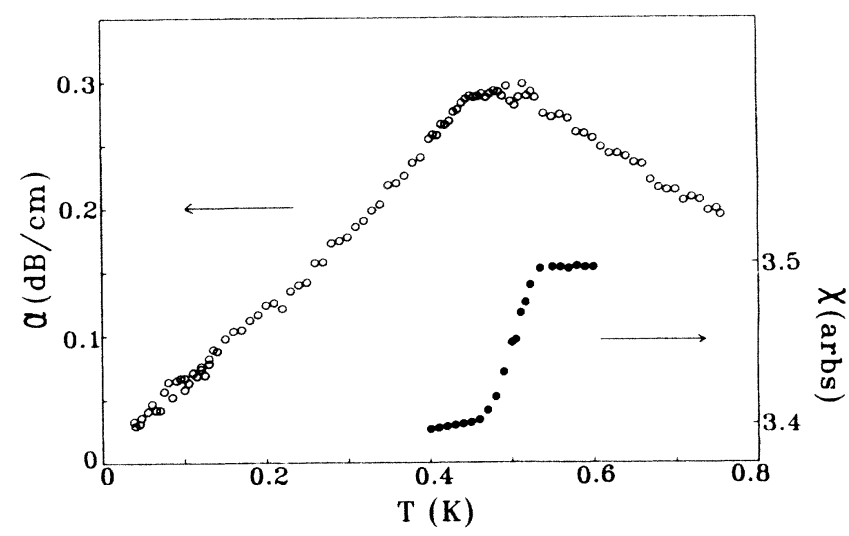

FIG. 1. Attenuation of transverse sound at $94 \mathrm{MHz}$ with $\hat{\mathbf{q}} \| \hat{\mathbf{b}}$ and $\hat{\mathbf{e}} \| \hat{\mathbf{a}}$, as measured from its extrapolated zerotemperature value. The ac susceptibility (right scale) is simultaneously measured through the superconducting transition. Note the linear dependence of the attenuation down to the lowest temperatures measured.

flatten off but continues to change even below $50 \mathrm{mK}$. This has not been seen previously. Second, there is no discontinuous change in the attenuation near $T_{c}$ as observed in type-I singlet superconductors. Third and last, there appears to be a maximum at a temperature slightly below $T_{c}$.

The attenuation in Fig. 1 is well fitted by a linear relation in temperature. In Fig. 2 the slope of this linear fit is shown as a function of the square of the frequency, $f^{2}$. The solid line is a linear least-squares fit required to go through the origin. It is clear from this figure that our measurements (even at the lowest temperatures) are in the hydrodynamic limit, ${ }^{3,12} q l<<1$. This result is also consistent with the absence of a jump in the attenuation at $T_{c}$. In the presence of impurities the shielding of the currents set up by the transverse sound due to the Meissner effect is washed out. Similar effects have been seen in impure type-I superconductors. ${ }^{8}$

We note here that the results shown in Fig. 1 represent the absolute value of the electronic contribution to the attenuation. However, the physical quantity of more fundamental interest is the viscosity, $\eta$, of the electrons and not the attenuation. These two quantities are related ${ }^{13}$ by the expression

$$
\alpha \sim \omega^{2} \eta / \rho v_{s}^{3},
$$

where $\omega=2 \pi f, \rho$ is the density of the solid, and $v_{s}$ the sound velocity. In our experiments we measure both the attenuation and the time-of-flight velocity simultaneously. Thus, we can evaluate the quantity $\alpha v_{s}^{3}$ which is proportional to the viscosity. This is shown in Fig. 3 for the case of the two polarizations at $f=132$ $\mathrm{MHz}$. The measured values of the velocities for the two cases are $(2.1 \pm 0.06) \times 10^{5} \mathrm{~cm} / \mathrm{s} \quad(\hat{\mathbf{e}} \| \hat{\mathbf{a}})$ and

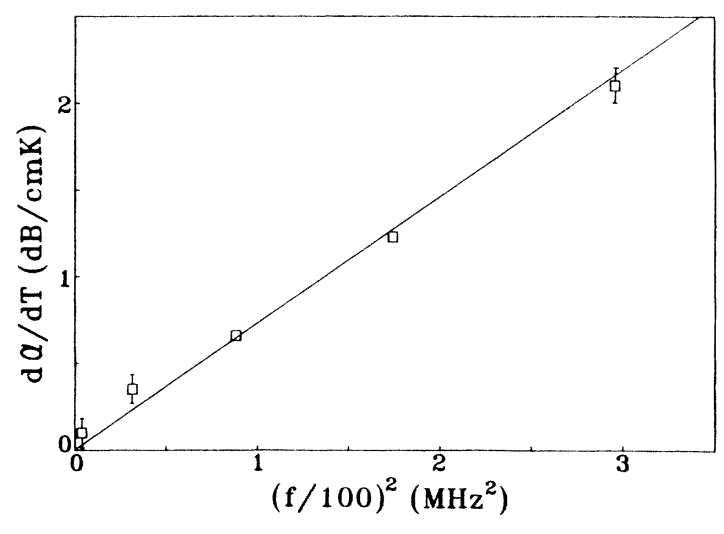

FIG. 2. The slope of the low-temperature attenuation of Fig. 1 plotted against the square of the frequency, indicating that our measurements are in the hydrodynamic regime.

$(1.4 \pm 0.03) \times 10^{5} \mathrm{~cm} / \mathrm{s}(\hat{\mathbf{e}} \| \hat{\mathbf{c}})$. It is immediately clear from Fig. 3 that the results for the two polarizations match in the normal state but are distinct in the superconducting phase. When analyzed in this fashion our results show that there is an enhancement of the attenuation for êlla over the values obtained in the superconducting state for $\hat{\mathbf{e}} \| \hat{\mathbf{c}}$.

A number of recent theories, 5,14 have been put forth in order to explain the ultrasonic attenuation in anisotropic superconductors. Calculations by Coppersmith and Klemm ${ }^{14}$ show that for a polar state the attenuation indeed does depend linearly on temperature. But these calculations are in the clean limit and should be inapplicable here in view of the results in Fig. 2. A theory by Pethick and Pines ${ }^{5}$ evaluates the ultrasonic attenuation and viscosity in anisotropic su-

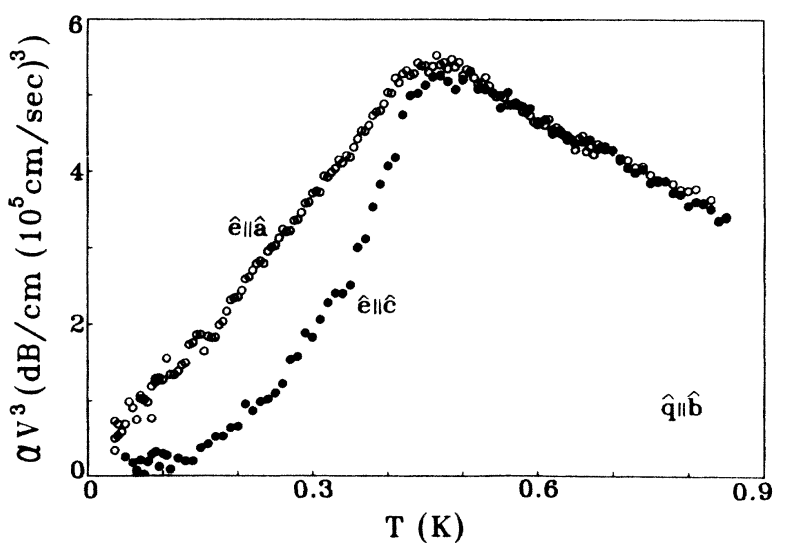

FIG. 3. $\alpha v_{s}^{3}$ (proportional to the viscosity) vs temperature at $132 \mathrm{MHz}$. The values for the two polarizations are identical in the normal state, but there is an enhanced viscosity in the superconductor for $\hat{\mathbf{e}} \| \hat{\mathbf{a}}$. 
perconductors where the dominant relaxation process is by impurity scattering. Their results indicate that in the weak-coupling limit, where the phase shifts due to impurity scattering are close to zero, the attenuation tends to a constant value. On the other hand, phase shifts in heavy-fermion systems are expected ${ }^{5}$ to be close to $\pi / 2$ in which case the attenuation should no longer be temperature independent. In this strongcoupling limit, Pethick and Pines calculate that the attenuation scales like $T^{2}$ for an ABM type of order parameter and like $T^{2} \ln ^{2} T$ for a polar-type order parameter. Hence, there is a crossover from a weakto a strong-coupling regime above a certain temperature $T_{1}$. For our results with $\hat{\mathbf{e}} \| \hat{\mathbf{c}}$ shown in Fig. 3 the attenuation is constant roughly below $T_{1} \sim 150 \mathrm{mK}$. Above this temperature our measurements are well described by either a $T^{2}$ of a $T^{2} \ln ^{2} T$ power law. Alternatively, if we consider the entire temperature range below $T_{c}$ we can fit our results with a $T^{3}$ power law better than either a $T^{2}$ or a $T^{2} \ln ^{2} T$. Similar observations have been made by Müller $e t$ al. in the case of longitudinal and transverse sound propagating along the $c$ axis. ${ }^{7}$ In this sense our results for the case $\hat{\mathbf{e}} \| \hat{\mathbf{c}}$ agree with their recent measurements, but are contrary to the $T^{2}$ dependence found by Bishop et al. ${ }^{3}$

We have a completely new result, however, for the sample with $\hat{\mathbf{e}} \| \mathrm{a}$ which we cannot understand within the framework of any of the aforementioned theories. Both our samples were cut from the same single crystal and were subjected to the same preparation procedures. That there are no differences between the two samples except for the plane of polarization is clear from the identical values of both $T_{c}$ and the viscosity in the normal state (Fig. 3). Recent experiments by Müller et al. ${ }^{15}$ in normal $\mathrm{UPt}_{3}$ also demonstrate the insensitivity of the transverse ultrasonic at tenuation to the orientation of the polarization.

It is unlikely that the different temperature dependences can be modeled by the assumption of only an anisotropic gap in the presence of nonmagnetic impurities. None of the present theories consider the scattering of sound by magnetic impurities or the spins which are confined to the basal plane. We speculate that the observed anisotropy is due to a coupling of sound to spin fluctuations in the superconducting phase. A similar coupling would be expected even in the normal phase, but in the absence of coherence between the spins the effect could be small and unobservable. Recent neutron-scattering experiments ${ }^{16}$ have revealed the importance of spin fluctuations and the Fermi-liquid nature of the excitations in $\mathrm{UPt}_{3}$. We also note that neither the experimentally determined values for thermal conductivity ${ }^{17}$ nor those for specific heat ${ }^{18}$ in the superconducting state are amenable to treatment by the theoretical models ${ }^{4,5,14}$ which we have considered above. It is possible that the anomalous behavior of these thermal properties of $\mathrm{UPt}_{3}$ below $T_{c}$ may in some way be related to the anomalous behavior of the ultrasonic attenuation.

In conclusion, we have performed the first measurements of ultrasonic attenuation in the basal plane of superconducting $\mathrm{UPt}_{3}$. For transverse sound with polarization parallel to the basal plane we find a linear temperature dependence of the attenuation coefficient between 35 and $400 \mathrm{mK}$. On the other hand, for polarization perpendicular to the basal plane our results are consistent with previous work and can be understood within the framework of available theories. Furthermore, the linear temperature dependence seems to arise from an additional attenuating mechanism not present for the perpendicular geometry. These facts call for additional theoretical efforts in an understanding of the propagation of sound in anisotropic superconductors in the dirty limit.

We should like to thank A. Auerbach, S. N. Coppersmith, G. Crabtree, A. J. Leggett, S. R. Nagel, J. P. Rodriguez, and especially D. Pines for helpful conversations. We appreciate the loan of Armstrong Adhesive from M. Levy. The work at Argonne National Laboratory was supported by the U. S. Department of Energy, Basic Energy Sciences Materials Sciences under Contract No. W-31-109-ENG-38. The work at the University of Chicago was supported by the National Science Foundation under Contract No. DMR 8351992, The Materials Research Laboratory, and a Joseph H. DeFrees Grant from Research Corporation. One of us (T.F.R.) acknowledges receipt of an Alfred P. Sloan Fellowship.

${ }^{1}$ G. R. Stewart, Z. Fisk, J. O. Willis, and D. L. Smith, Phys. Rev. Lett. 52, 679 (1984).

2J. W. Chen, S. E. Lambert, M. B. Maple, Z. Fisk, J. L. Smith, G. R. Stewart, and J. O. Willis, Phys. Rev. B 30, 1583 (1984); C. M. Varma, unpublished; K. Scharnberg and R. A. Klemm, Phys. Rev. Lett. 54, 2445 (1985).

${ }^{3}$ D. J. Bishop, C. M. Varma, B. Batlogg, E. Bucher, Z. Fisk, and J. L. Smith, Phys. Rev. Lett. 53, 1009 (1984).

${ }^{4}$ J. P. Rodriguez, Phys. Rev. Lett. 55, 250 (1985).

${ }^{5}$ C. J. Pethick and D. Pines, to be published.

${ }^{6} \mathrm{~K}$. Maki, in Superconductivity, edited by R. D. Parks (Dekker, New York, 1969), Vol. 2, p. 1035.

${ }^{7}$ V. Müller, D. Maurer, E. W. Scheidt, Ch. Roth, K. Luders, E. Bucher, and H. E. Bömmel, to be published.

${ }^{8}$ J. A. Rayne and C. K. Jones, in Physical Acoustics; Principles and Methods, edited by W. P. Mason and R. N. Thurston (Academic, New York, 1981), Vol. 7, p. 149; J. R. Lebowitz, Phys. Rev. A 133, 84 (1964).

${ }^{9} \mathrm{~J}$. P. Rodriguez, private communication.

${ }^{10}$ Armstrong Adhesives, Ellsworth Systems, Milwaukee, Wisconsin.

${ }^{11}$ Y. H. Jeong, S. R. Nagel, and S. Bhattacharya, to be pub- 
lished.

${ }^{12}$ A. B. Pippard, Philos. Mag. 46, 1104 (1955).

${ }^{13}$ W. P. Mason, Phys. Rev. 97, 557 (1955).

${ }^{14} \mathrm{~S}$. N. Coppersmith and R. Klemm, to be published.

${ }^{15}$ V. Müller, D. Maurer, K. de Groot, E. Bucher, and H. E. Bömmel, Phys. Rev. Lett. 56, 248 (1986).
${ }^{16}$ G. Aeppli, E. Bucher, and G. Shirane, Phys. Rev. B 32, 7579 (1985).

${ }^{17}$ D. Jaccard, J. Floquet, P. Lejay, and J. L. Tholence, J. Appl. Phys. 57, 3082 (1985).

${ }^{18}$ A. Sulpice, P. Gandit, J. Chaussy, J. Floquet, D. Jaccard, and P. Lejay, to be published. 